$\mathrm{p}=0.260$ ) and absolute counts ( 10 vs 5 cells $/ \mu \mathrm{L} ; \mathrm{p}=0.343$ ) compared to anti-SSAnegative patients.

Although CD24 ${ }^{\text {hi }} \mathrm{CD} 27^{+}$B-cells frequencies and absolute counts did not differ between patients with active $(n=27)$ or inactive disease $(n=30),(16.9 \%$ vs $17.0 \%$, $p=0.946 ; 25$ vs 31 cells $/ \mu l, p=0.179$ ), patients with higher disease activity (ESS DAl $\geq 5)(n=9)$ presented lower absolute counts of CD24 ${ }^{\text {hi }} \mathrm{CD} 27^{+}$B-cells (18 vs 31 cells $/ \mu \mathrm{L}, \mathrm{p}=0.096$ ) and lower CD24 ${ }^{\text {hi }} \mathrm{CD} 38^{\text {hi }} \mathrm{B}$-cells (4 vs 10 cells $/ \mu \mathrm{L}, \mathrm{p}=0.075$ ), and higher Th1/Breg CD24 ${ }^{\text {hi }} \mathrm{CD} 27^{+}$ratios, (16.2 vs 9.2, $\mathrm{p}=0.064$ ).

Considering all patients, a negative correlation was found between the ESSDAI score and the absolute numbers of either $\mathrm{CD} 24^{\mathrm{hi}} \mathrm{CD} 27^{+}$B-cells $(r=-0.277$; $\mathrm{p}=0.037)$ and Tregs $(\mathrm{r}=-0.311 ; \mathrm{p}=0.019)$.

Correlations with ESSDAI was stronger when looking at patients with ESSDAI $\geq 5$ : for the percentages of $C D 24{ }^{\text {hi }} C D 27^{+} B$-cells, $r=-0.705, p=0.023$; for $C D 24{ }^{\text {hi }} C D 27^{+}$ B-cells absolute counts, $r=-0.644 ; p=0.045$; and for the absolute counts of Tregs, $r=-0.862 ; p=0.001$

Using ROC curves to discriminate pSS from HC, better AUCs were obtained for CD24 ${ }^{\text {hi }} \mathrm{CD} 27^{+}$Breg cells (cut-off 34 cells $/ \mu \mathrm{L}, \mathrm{AUC}=0.81$ ), Tregs $/ \mathrm{CD} 24^{\text {hi }} \mathrm{CD} 27^{+}$ Breg ratio (cut-off 1.98, $\mathrm{AUC}=0.74$ ) and $\mathrm{T}_{\mathrm{h}} 1 / \mathrm{CD} 24^{\text {hi }} \mathrm{CD} 27^{+}$Breg ratio (cut-off 12.23, $\mathrm{AUC}=0.70$ ), corresponding to a specificity for pSS of $0.83,0.75$ and 0.70 , respectively, and sensitivity of $0.75,0.72$ and 0.67 , respectively.

In pSS, lower CD2 $4{ }^{\text {hi }} \mathrm{CD} 27^{+}$B-cell counts, as well as higher Tregs $/ C D 24^{\text {hi }} \mathrm{CD} 27^{+}$ Breg and $T_{h} 1 / C D 24{ }^{h i} C D 27^{+}$Breg ratios, were associated to a higher frequency of autoantibodies and higher gammaglobulin.

Conclusion: Our findings demonstrated a significant decrease in the Bregenriched $\mathrm{CD} 24^{\mathrm{hi}} \mathrm{CD} 27^{+} \mathrm{B}$-cell subset in pSS, which presented a negative correlation with the disease activity. We have demonstrated significant differences in the $\mathrm{CD} 24^{\mathrm{hi}} \mathrm{CD} 27^{+} \mathrm{B}$-cell subset and respective ratios, presenting a good discriminatory capacity compared to $\mathrm{HC}$. Therefore, this subset may have diagnostic utility in pSS, as it may support the presence of immune dysregulation in suspected cases that don't fulfil the pSS classification criteria. Further studies with increased number of samples and a prospective design are needed to explore this hypothesis.

Disclosure of Interests: Filipe Barcelos Consultant for: Pfizer; Ely-Lilly, Speakers bureau: Novartis, Catarina Martins: None declared, Carlos Geraldes: None declared, Ana Luísa Papoila: None declared, Joana Cardigos: None declared Glória Nunes: None declared, Teresa Lopes: None declared, Nuno Alves: None declared, José Vaz-Patto: None declared, Jaime Branco: None declared, Luís Miguel Borrego Grant/research support from: MSD, Consultant for: MSD; Tecnifar, Paid instructor for: MSD; AstraZeneca, Speakers bureau: MSD; Tecnifar; AstraZeneca

DOI: 10.1136/annrheumdis-2019-eular.2614

\section{AB0162 IMPLEMENTATION OF HYDROXYCHLOROQUINE AT THE PRE-CONCEPTIONAL STAGE IN THE TREATMENT OF ANTIPHOSPOLIPID SYNDROME ASSOCIATED WITH SYSTEMIC LUPUS ERYTHEMATOSUS}

Dmitrii Bublikov, Marina Nikolaeva. Altay State Medical University, Barnaul, Russian Federation

Background: The problem of correcting the antiphospholipid syndrome (APS) at the planning stage of pregnancy of patients with systemic lupus erythematosus (SLE) is an important task of rheumatology.

Objectives: To study the effect of hydroxychloroquine $(\mathrm{HC})$ on the level of antiphospholipid antibodies titer in women with SLE of low degree of activity at the planning stage of pregnancy.

Methods: The study included 7 women aged from 18 to 44 years with a definite diagnosis of low-level SLE (1-5 points according to the SLEDAI2K scale) and confirmed APS. All patients were characterized by a history of three or more reproductive losses in the gestational age of 10 weeks or more. According to standard laboratory tests, the level of antibodies to cardiolipin ( $/ g M / / g G)$ and/or to $\beta 2$-glycoprotein I (IgM/lgG) ( $\beta 2 \mathrm{GPI})$ in a titer above 99 percentile was determined twice in a 12-week interval. Before inclusion in the study, patients received methylprednisolone and acetylsalicylic acid in the recommended dosages. Other drugs potentially affecting the activity of SLE or hemostasis indicators of the patient during the observation period were not administered. At the time of inclusion, patients were prescribed with hydroxychloroquine $(\mathrm{HC})$ in a dose of $400 \mathrm{mg}$ per day orally, in addition to the therapy received earlier. The titer of antibodies to cardiolipin (IgM/ $\lg G$ ) and antibodies to $\beta 2 \mathrm{GPI}(\lg M / \lg G$ ) was determined three times: initially, after 3 and 6 months from the start of therapy. Statistical data processing was performed using the MS Excel 2010 statistical software package and MedCalc Version 17.9.7. Data of laboratory parameters are presented in the form of median (Me) and $95 \%$ confidence interval $(95 \% \mathrm{Cl})$. Comparison of dependent samples was performed using non-parametric methods (Wilcoxon Signed Ranks Test). The statistical significance of the hypotheses was accepted at a level of $p<0.05$ Results: The initial SLEDAI2K index constituted $4.0 \pm 1.0$ points. The median of the level of antibodies to cardiolipin is defined as 69.2 [95\% Cl $57.4-80.0] \mathrm{U} / \mathrm{ml}$., antibodies to $\beta 2 \mathrm{GPI}$ - 69.24 [95\% Cl 49.7 - 87.5] U/ml. After 3 and 6 months from the start of $\mathrm{HC}$ administration, the SLEDAI2K index did not statistically significantly change $(p=0.09)$. 3 months later, a statistically significant decrease in the level of antibodies to cardiolipin ( $/ g M / l g G$ ) by $30.4 \%$ was determined relative to the initial one [Me $21.43(95 \% \mathrm{Cl} 20.2-46.1), \mathrm{p}=0.001]$. By the 6 th month of ther apy, a reduced level of antibodies to cardiolipin remained unchanged; there was no statistically significant difference between the rates after 3 and 6 months of HC therapy. The level of antibodies to $32 \mathrm{GPI}(\mathrm{lgM} / \mathrm{lg} G)$ after three months from the start of therapy remained at the level of the indicator before inclusion in the study. A statistically significant decrease in the level of antibodies to 32 GPI by $28.5 \%$ compared to the initial one was determined 6 months after the start of $\mathrm{HC}$ adminis tration [Me 19.17 (95\% Cl 10.8-27.4), $\mathrm{p}=0.003$ ].

Conclusion: The study showed that, against the background of additional administration of hydroxychloroquine in patients with APS with low-level SLE, a decrease in the level of antibodies to cardiolipin $(/ g M / / g G)$ by $30.4 \%(p=0.001)$ was observed after 3 months of therapy, to $\beta 2$-glycoprotein I ( $\lg M / \lg G$ ) - by $28.5 \%$ $(p=0.003)$ after 6 months of therapy.

Disclosure of Interests: None declared

DOI: 10.1136/annrheumdis-2019-eular.4937

\section{AB0163 THE ROLE OF IMMUNOREGULATORY MOLECULES ON FATIGUE IN PRIMARY SJÖGREN'S SYNDROME}

Kallpana Dhas ${ }^{1}$, Emmanuella Traianos ${ }^{2}$, Jessica Tarn $^{2}$, Wan Fai $\mathrm{Ng}^{2} .{ }^{1}$ School of Medical Education, Newcastle University, Newcastle upon Tyne, United Kingdom; ${ }^{2}$ Institute of Cellular Medicine, Newcastle University, Newcastle upon Tyne, United Kingdom

Background: Primary Sjögren's syndrome (pSS) is a chronic autoimmune rheu matic disease characterized by exocrine gland dysfunction. The clinical presentation of pSS can vary considerably from predominantly 'sicca' symptoms such as dry eyes and dry mouth to systemic manifestations such as arthralgia, vasculitis and fatigue ${ }^{1,2}$. Previous work from our laboratory has suggested that immunoregulatory pathways might play a role in fatigue development in patients with $\mathrm{pSS}^{3}$. Objectives: The first aim was to measure the serum levels of three candidate immunoregulatory molecules (melatonin, TGF- $\beta$ and IL-10) and determine any relationship with fatigue severity. The second aim was to compare molecular pro files of the immunoregulatory mediators between pSS patients and healthy controls. The third aim was to identify key predictors of fatigue in pSS.

Methods: Serum levels of the three molecules were measured in 124 patients with pSS and 28 healthy non-fatigued controls selected from the United Kingdom Primary Sjögren's Syndrome Registry using various assays. IL-10 concentrations were measured using a cytometric bead array-based immunoassay, melatonin levels were determined using ELISA and TGF- $\beta$ concentrations were quantified using a bioassay in which HKC-8 cells were stably transfected with a TGF- $\beta$ inducible CAGA-luciferase reporter construct. Patient fatigue levels were evaluated with a validated self-complete questionnaire and the scores were compared with the immunoregulatory molecular levels using analysis of variance. Significance in immunoregulatory mediators between the patients and controls was determined using the Wilcoxon test. Ordinal logistic regression analysis was performed in a smaller subset of patients $(N=75)$ to identify the key predictors of fatigue in pSS

Results: IL-10 was significantly higher in the sera of patients with pSS compared to the healthy controls $(p<0.0001)$. Melatonin showed a positive correlation with fatigue levels within the patient cohort $(\rho=0.1664)$ whereas IL-10 and TGF- $\beta$ were inversely related to fatigue severity $(\rho=0.1265$ and $\rho=0.0643$ respectively). The regression model used the three investigated immunoregulatory molecules, disease specific, haematological and clinical parameters as well as patient reported depression, anxiety and pain as predictors. The model was able to predict fatigue levels to a $67 \%$ accuracy.

Conclusion: The study suggests that melatonin may play a role in regulating the immune response in pSS and may affect fatigue levels in patients. Dryness, anxiety, pain and melatonin appear to be the most powerful predictors of fatigue in pSS. Further research into the effects of these immunoregulatory molecules is necessary to gain a better understanding of the pathophysiology of fatigue in pSS

\section{REFERENCES}

[1] Shahane A, Patel R. The epidemiology of Sjogren's syndrome. Clinical Epidemiology. 2014;6:247-255

[2] $\mathrm{Ng} \mathrm{W}$, Bowman S. Primary Sjogren's syndrome: too dry and too tired. Rheumatology. 2010;49(5):844-853.

[3] Howard Tripp N, Tarn J, Natasari A, et al. Fatigue in primary Sjögren's syndrome is associated with lower levels of proinflammatory cytokines RMD Open. 2016;2(2):e000282.

Disclosure of Interests: None declared

DOI: 10.1136/annrheumdis-2019-eular.4815 


$\mathrm{AB} 0164$

\section{THE EXPRESSION AND CLINICAL SIGNIFICANCE OF DIFFERENT FORMS OF LILRA3 IN SYSTEMIC LUPUS ERYTHEMATOSUS}

Yan Du, Meiju Zhou, Xinyu Wu, Jing Xue, Huaxiang Wu, Yujie Jiang. Zhejiang university school of medicine second affiliated hospital, Hangzhou, China

Background: LILRA3 is a member of the LILR family produced as a soluble molecule by monocytes and macrophages. A 6.7-kb deletion in the gene of LILRA3 results in a null allele and an absence of function. The frequencies of the $6.7-\mathrm{kb}$ deletion vary greatly in different populations, considerably higher in Northeastern Asians (0.56-0.84) compared with Africans (0.10) or Europeans (0.17). The homozygous LILRA3 deletion (nonfunctional LILRA3) has been demonstrated to be associated with Sjogren's syndrome (SS) and multiple sclerosis (MS) in Caucasians. However, in our previous studies, we found that in Chinese Han population, the LILRA3 non-deletion (namely functional LILRA3) contributes to susceptibility and subphenotypes of SLE and SS. With respect to the significant role of LILRA3 in immune-modulatory functions, we studied the role of LILRA3 in SLE.

Objectives: Our previous study has shown that functional LILRA3 contributes to susceptibility and subphenotypes of systemic lupus erythematosus (SLE). However, the mechanism remains unclear. We aimed to evaluate the role of LILRA3 in SLE.

Methods: 126 SLE patients and 48 healthy controls were recruited in this study. Functional studies were performed using intracellular flow cytometry and ELISA. Results: Both LILRA3 levels in serum and $\mathrm{CD} 14^{+}$monocytes were significantly elevated in SLE patients compared with healthy controls. Elevated LILRA3 level was found positively correlated with SLEDAI. Furthermore, more elevated LILRA3 levels were found in patients with higher SLEDAI, presence of lupus nephritis and thrombocytopenia.

Conclusion: Both LILRA3 levels in serum and CD14+ monocytes significantly increased in SLE and positively correlated with disease activity and severity. The up-regulation of LILRA3 expression may serve as a biomarker of disease activity and severity of SLE.

Table 1. Correlations of LILRA3 levels with the studied parameters in SLE patients

\begin{tabular}{lcccc}
\hline Clinical manifestations & \multicolumn{2}{c}{ LILRA3 in monocytes (MFI) } & \multicolumn{2}{c}{ LILRA3 in serum (pg/mL) } \\
& Spearman's $r$ & $P$ value & Spearman's $r$ & $P$ value \\
\hline SLEDAI & $\mathbf{0 . 3 9 8}$ & $\mathbf{0 . 0 0 1 8 ^ { * * }}$ & $\mathbf{0 . 2 5 7}$ & $\mathbf{0 . 0 0 3 6 ^ { * * }}$ \\
24h proteinuria excretion & $\mathbf{0 . 3 2 8}$ & $\mathbf{0 . 0 2 4 ^ { * }}$ & 0.159 & 0.09 \\
Leucocytes & -0.033 & 0.680 & -0.253 & $\mathbf{0 . 0 0 3 7 ^ { * * }}$ \\
Thrombocytes & -0.176 & 0.213 & -0.144 & 0.11 \\
Anti-dsDNA Ab & 0.066 & 0.627 & -0.061 & 0.498 \\
C3 & -0.138 & 0.290 & -0.076 & 0.392 \\
C4 & -0.140 & 0.282 & -0.025 & 0.777 \\
IgA & 0.068 & 0.604 & 0.017 & 0.852 \\
IgM & 0.107 & 0.417 & 0.053 & 0.557 \\
IgG & 0.143 & 0.218 & 0.228 & $0.01^{*}$ \\
CRP & -0.164 & 0.237 & 0.088 & 0.339 \\
\hline
\end{tabular}

SLE: systemic lupus erythematosus; SLEDAI: systemic lupus erythematosus disease activity index; LILRA3: Leukocyte immunoglobulin-like receptor A3; ANA: antinuclear antibody; AntidsDNA Ab: anti-double strand DNA Antibody; ACL: anticardiolipin antibody; AnuA:

antinucleosome antibody; Sm: anti-Smith antibody; SS: anti-SSA antibody; C3 Complement component 3; C4: Complement component 4. CRP: C-reactive protein. ${ }^{\star} P<0.05,{ }^{\star *} P<$

01. Spearman's correlation coefficient $(r)$ was applied to detect correlation between two types of numerical data.

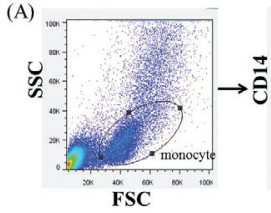

(B)

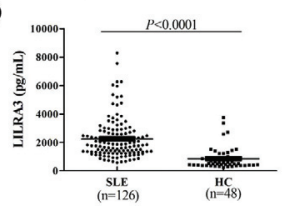

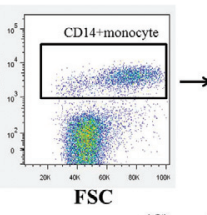

$(\mathrm{C})$

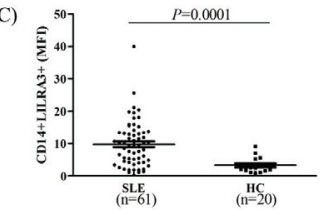

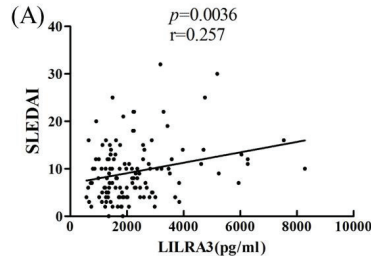
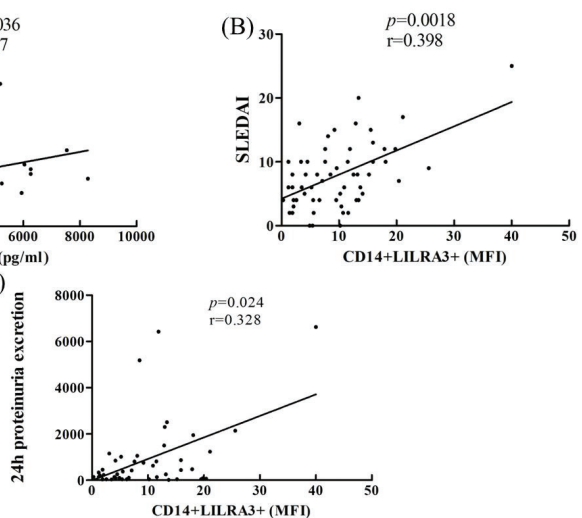

Figure 2
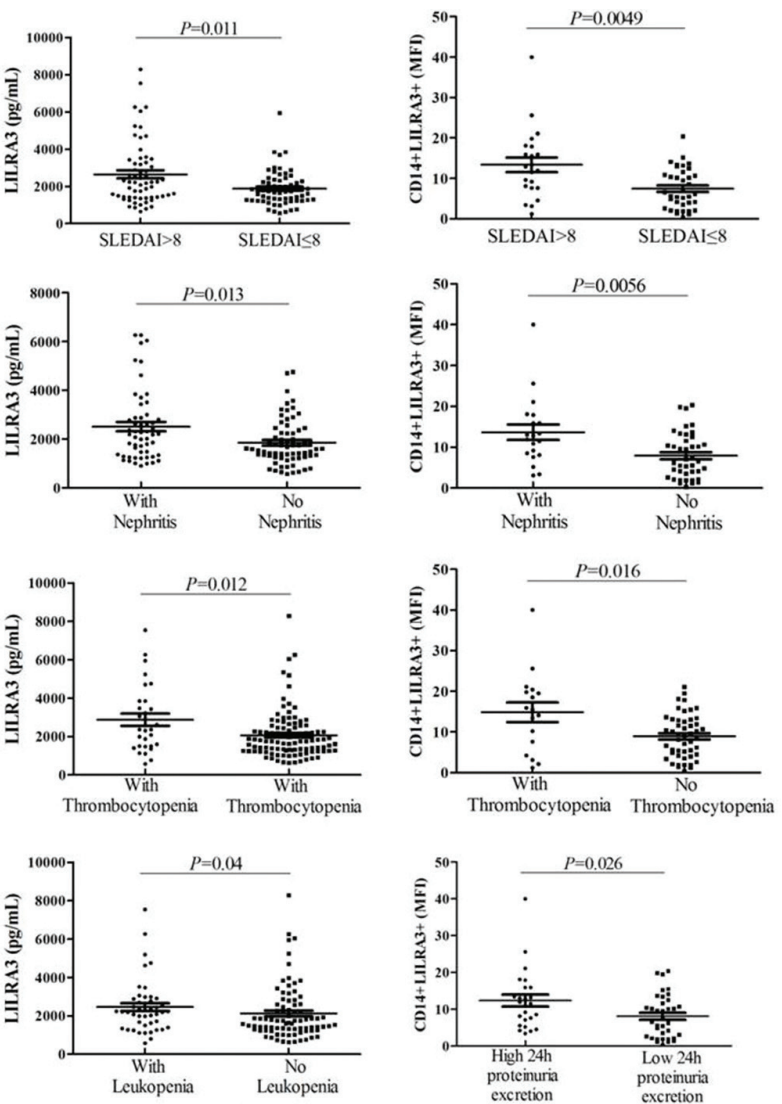

(A)

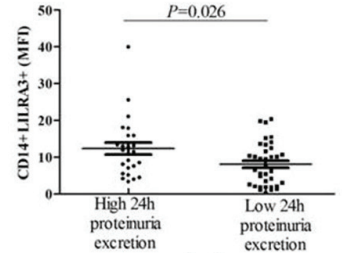

(B)
Figure 1

Figure 3

\section{REFERENCES}

[1] Du, Y., Y. Su, J. He, Y. Yang, Y. Shi, Y. Cui, C. Luo, X. Wu, X. Liu, F. Hu, X. Ma, L. Zheng, J. Zhang, X. Zuo, Y. Sheng, L. Wu, X. Zhang, J. Guo, and $\mathrm{Z}$. $\mathrm{Li}$, Impact of the leucocyte immunoglobulin-like receptor $A 3$ (LILRA3) on susceptibility and subphenotypes of systemic lupus erythematosus and Sjogren's syndrome. Ann Rheum Dis, 2015. 74(11): p. 2070-5.

Disclosure of Interests: None declared

DOI: 10.1136/annrheumdis-2019-eular.2418 